\title{
Study of DC Breakdown in Multilayered Insulation Systems
}

\author{
Miao Hao ${ }^{1}$, Yunpeng Zhan ${ }^{1}$, George $\mathrm{Chen}^{1}$, Lu Pu${ }^{2}$, Xuefeng Zhao ${ }^{2}$, Wei Duan ${ }^{2}$, Haofei Sun ${ }^{2}$, Zeli Ju ${ }^{2}$, Nan Wang ${ }^{2}$ \\ ${ }^{1}$. School of Electronics and Computer Science, University of Southampton, Southampton, UK \\ ${ }^{2}$. State Grid Shaanxi Electric Power Research Institute, Xi'an, China
}

\begin{abstract}
Multilayer insulation systems have been widely used in both high voltage alternating current (HVAC) and high voltage direct current (HVDC) power systems. A well designed multilayer insulation structure could benefit the performances and reliability of the overall insulation system and as well as costs reduction. In this present work, DC breakdown strengths of various combinations of multilayer polyethylene terephthalate (PET) films (from one to four layers) with a same total thickness were investigated. It has been found that multilayer symmetrical structures can enhance the breakdown strength of the insulation system. Space charge characteristics of these various configurations have been initially studied, in order to understand the results of the DC breakdown tests. The space charge results indicate that large amount of fast moving charges presences immediately after the voltage application, which can be partially blocked and trapped by the interfaces between film layers. The trapped charges could further distort the electric field distributions in the insulation system, and resulting field enhancement in thin layers, particularly in unsymmetrical configurations. However, more evidences and investigations are required for better understanding the mechanisms of breakdown in multilayer insulation system.
\end{abstract}

\section{INTRODUCTION}

In recent years, the rapid development HVDC technologies greatly benefits the capability and reliability of modern power networks. The development of novel and high performance dielectric materials has attracted abundant research interests, which enables the new generation of HVDC power equipment with higher operating voltage, larger capacity and reasonably low costs [1]. It has been recognized, in addition to insulation properties, the structure of an insulation system plays a vital role in the performance of the insulation system [2].

Multilayered insulation system have been widely applied for many decades in $\mathrm{HV}$ power equipment, such as power transformers and power cables. The optimized insulation structure could improve the mechanical and thermal properties without losing, sometimes even enhancing, electrical properties of the whole insulation system. It has been reported that the multilayer polymeric films with a same total thickness enhances the $\mathrm{AC}$ breakdown strength compared with a single layer film with the same thickness [3]. However, for the applications of HVDC technologies, the breakdown and failure mechanism could be different from those for HVAC technologies, for example, the presence and accumulation of space charges under HVDC electric fields have been a major concern for the early breakdown of dielectric materials and insulation systems [4-6]. Therefore, in order to achieve the optimized insulation structure for HVDC applications, it is very important to investigate the relationships between multilayered insulation structures and their electrical properties.

In this present paper, the DC breakdown strengths of various combinations of multilayer PET films (from one to four layers) with a same total thickness were investigated. To understand the mechanism behind the different breakdown strengths, space charge characteristics of these combinations were studied.

\section{EXPERIMENTAL SETUP}

Commercial PET films with four different thicknesses were used as samples in this work, including 25, 50, 75 and 100 $\mu \mathrm{m}$. The configuration of samples are shown in Table 1. Total thickness of all the combinations was equal to $100 \mu \mathrm{m}$. The film samples were cut into $5 \mathrm{~cm} \times 5 \mathrm{~cm}$ for both the breakdown and space charge measurements.

TABLE 1

PET Film Sample Configurations

\begin{tabular}{|c|c|}
\hline Layer number & Sample Configuration with thickness \\
\hline 1 & $100 \mu \mathrm{m}$ \\
\hline 2 & $75+25 \mu \mathrm{m}$ \\
\hline 2 & $50+50 \mu \mathrm{m}$ \\
\hline 3 & $25+50+25 \mu \mathrm{m}$ \\
\hline 4 & $25+25+25+25 \mu \mathrm{m}$ \\
\hline
\end{tabular}

For the DC breakdown strength measurements, samples were placed between two $6.3 \mathrm{~mm}$ in diameter ball bearing electrodes in silicone fluid (Dow Corning 200/20CS) at the room temperature. A ramping voltage was applied with a rate of $100 \mathrm{~V} / \mathrm{s}$ until the sample failure. For each sample configuration, the breakdown test repeated no less than twenty times and the ball bearing electrodes were changed after 10 tests to avoid electrode pitting. The breakdown results were analyzed by using Weibull distribution.

Space charge characteristics of samples were measured by using pulsed electroacoustic (PEA) technique. In this work, a pulse voltage of $600 \mathrm{~V}$ with a pulse width of $5 \mathrm{~ns}$ was applied. The top electrode (anode in this work) is semiconductive layer, while the bottom electrode (cathode in this work) is aluminum plate. The sensor thickness of the PEA system is 9 $\mu \mathrm{m}$. Samples were placed on the bottom electrode and firmly pressed by the top electrode to achieve a good acoustic contact. The measurement protocol includes: (i) Volts-on test: 
an $1.8 \mathrm{kV}(18 \mathrm{kV} / \mathrm{mm})$ constant DC voltage was applied to the sample for a period of 1 hour; (ii) Decay test, after the volt-on test, the DC voltage was removed to allow the accumulated space charges decay for a period of 1 hour. For each sample configuration, more than three measurements were repeated and all the measurements were carried out at room temperature.

\section{RESULTS AND DISCUSSIONS}

\section{A. DC Breakdown Results}

The data of breakdown tests for each sample configuration were statistically analyzed with two-parameter Weibull distribution [7]. The results show that the DC breakdown strength changes depending on the configurations of samples. Figure 1 gives an example of the breakdown strength of a single layer $100 \mu \mathrm{m}$ sample and a four-layer $25 \mu \mathrm{m}$ sample. It shows an increase in the breakdown strength from 470.2 $\mathrm{kV} / \mathrm{mm}$ (single layer) to $541.3 \mathrm{kV} / \mathrm{mm}$ (four-layer). Table 2 summarises the Weibull parameters of DC breakdown characteristics for all configurations, where $\alpha$ is the breakdown strength at the cumulative failure probability of $63.2 \%$, and $\beta$ is the shape parameter which represents the spread of the data.

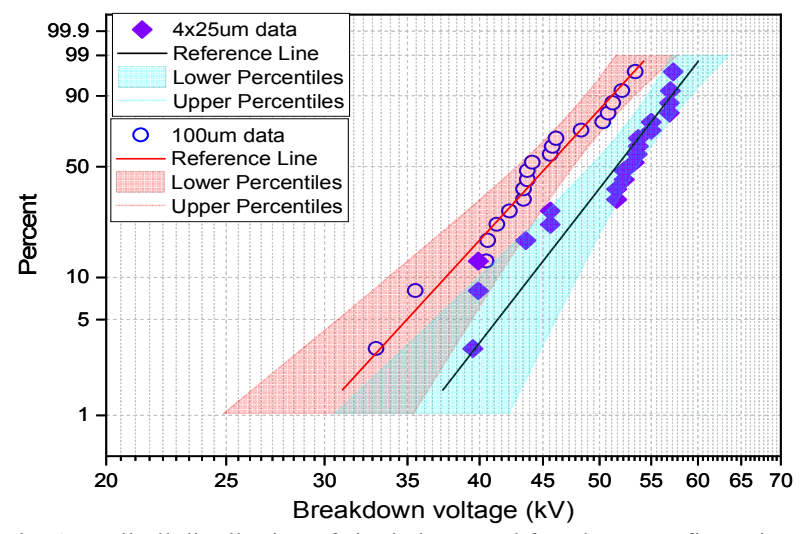

Fig. 1. Weibull distribution of single layer and four-layer configurations (Confidence Intervals 95\%).

TABLE 2

Weibull Distribution Parameters for 5 Configurations

\begin{tabular}{|c|c|c|}
\hline Sample Configuration & $\alpha(\mathrm{kV} / \mathrm{mm})$ & $\beta$ \\
\hline Single layer $100 \mu \mathrm{m}$ & 470.24 & 10.0 \\
\hline Two-layer $75+25 \mu \mathrm{m}$ & 478.62 & 10.0 \\
\hline Two-layer $50+50 \mu \mathrm{m}$ & 496.40 & 13.2 \\
\hline Three-layer $25+50+25 \mu \mathrm{m}$ & 535.64 & 16.7 \\
\hline Four-layer $25+25+25+25 \mu \mathrm{m}$ & 541.3 & 12.7 \\
\hline
\end{tabular}

Based on the results shown in Table 2, it can be found that (i) The breakdown strengths of the single layer sample and two-layer sample with unsymmetrical configuration are lowest. (ii) The breakdown strength increases with the increasing layers. (iii) For the same layer number, the symmetrical configuration could enhance the breakdown strength. (iv) The configuration with more layers and symmetric structure shows the highest breakdown strength.

\section{B. Effects of Interfaces}

In multilayer insulation system, interfaces usually play a vital role in electrical performance of the insulation system. It has been reported that interface can block the charge movement as a barrier in both homogenous dielectric materials and inhomogeneous dielectric materials. In the present work, it is interesting to note that little space charge accumulation can be observed in the single layer $100 \mu \mathrm{m}$ PET sample. However, space charge accumulation can be clearly observed in the multilayer configurations, as shown in Figure 2 (single layer vs. three-layer as an example). This may suggest that a considerable amount of fast mobile charges exist within the single layer sample, which cannot be detected by the PEA system as they are not "trapped". Once the interfaces are introduced, these mobile charges will be blocked and trapped in the region adjacent to the interfaces and become detectable. Similar phenomena in polyethylene have been reported in [8]. The large amount of fast moving charges within the single layer sample could potentially result in the low breakdown strength.

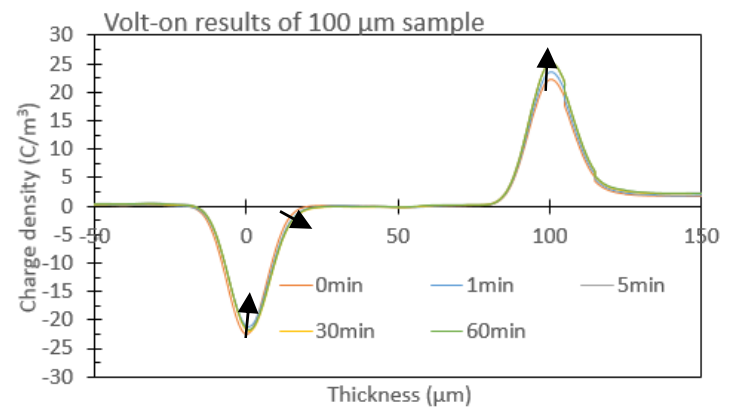

(a)

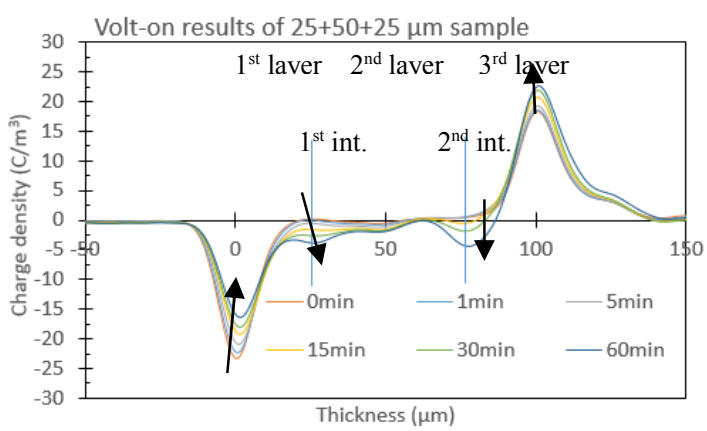

(b)

Fig. 2. Space charge profiles during volts-on in (a) single layer sample and (b) three-layer sample.

\section{Effects of unsymmetrical configuration}

Due to the charge barrier effect caused by the presence of interfaces, space charges accumulate at the interfacial region. When the configuration is unsymmetrical, for example, $75+$ $25 \mu \mathrm{m}$, the accumulated charges could enhance the electric field distribution in the thinner layer, which could leading to an early failure. Figures 3 (a) and (b) show the accumulated charges within the $75+25 \mu \mathrm{m}$ configuration sample after the 1 hour voltage application. In this configuration, the layer of $25 \mu \mathrm{m}$ was in contact with the anode (which is semiconductive layer) during the volts-on process. It can be seen that large amount of negative charges accumulated at the interfacial 
region, which behaves as heterocharges during the voltage application period. Therefore, the electric field within the 25 $\mu \mathrm{m}$ layer is greatly enhanced, as shown in Figure 3 (c). If the applied voltage is very high (e.g. in the breakdown test), this electric field enhancement could be significant and initiates breakdown process, resulting in a low breakdown strength.

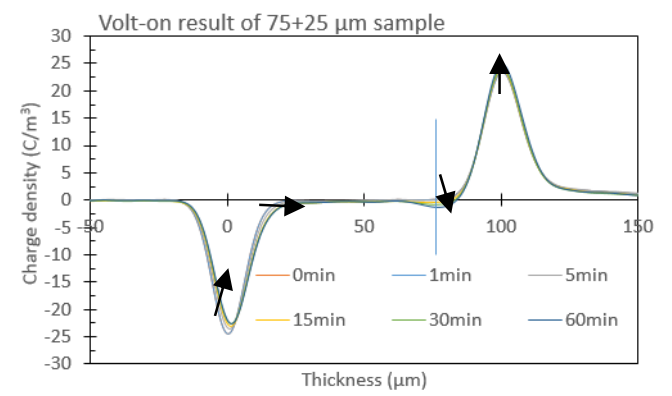

(a)

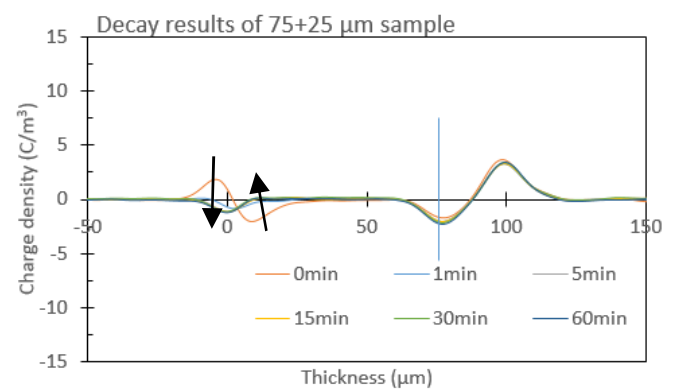

(b)

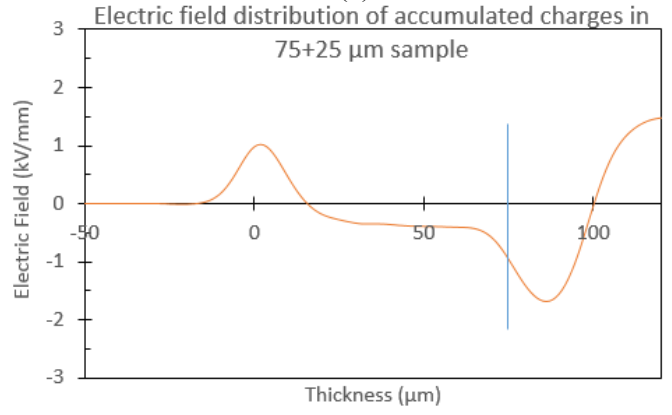

(c)

Fig. 3. Space charge profiles in unsymmetrical configuration (a) volt-on (b) decay and (c) corresponding electric field distribution of accumulated charges.

Moreover, as shown in Figure 3 (b), considerable amount of negative charges located in the region adjacent to the cathode can be observed shortly after the voltage removal. However, these charges dissipate very fast, i.e. disappear within 1 minute. On the other hand, the charges trapped at the interface can hardly dissipate. This phenomena also suggests that (i) large amount of fast moving charges exist within the PET film; (ii) the presence of interface can hinder the movement of these charges through the whole insulation system and trap them deeply in the interfacial region.

\section{Effects of symmetrical configuration}

Space charge profiles in the three symmetrical configurations $(50+50 \mu \mathrm{m}, 25+50+25 \mu \mathrm{m}$ and $25+25+25+25 \mu \mathrm{m})$ are shown in Figure 4.
As shown in Figure 4 (a), the presence of the interface in the middle of the insulation system can prevent both positive and negative charges propagate through the system. Therefore, negative charges accumulate in the layer contacted with the cathode and positive charges accumulate in the layer contacted with the anode next to the interface. Based on the space charge profiles, the electric field will be enhanced in the interfacial region.

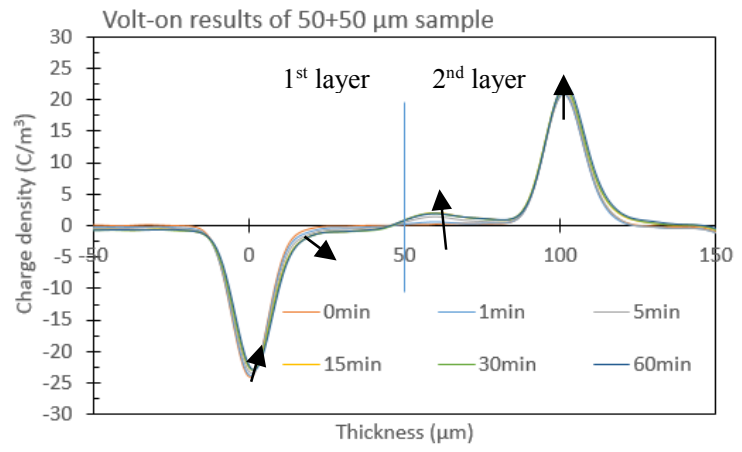

(a)

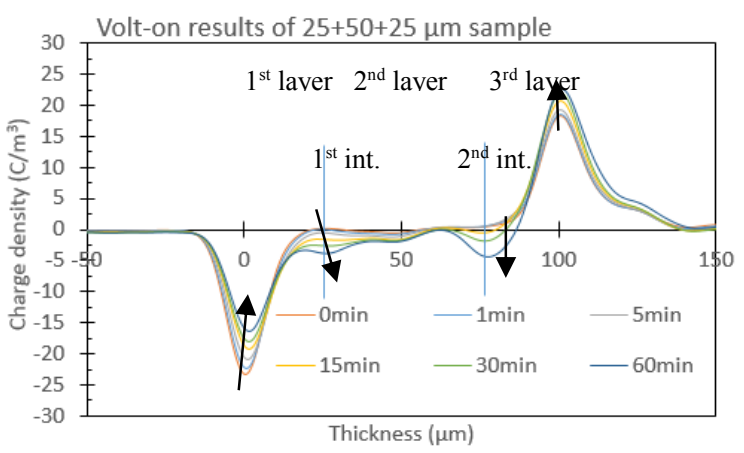

(b)

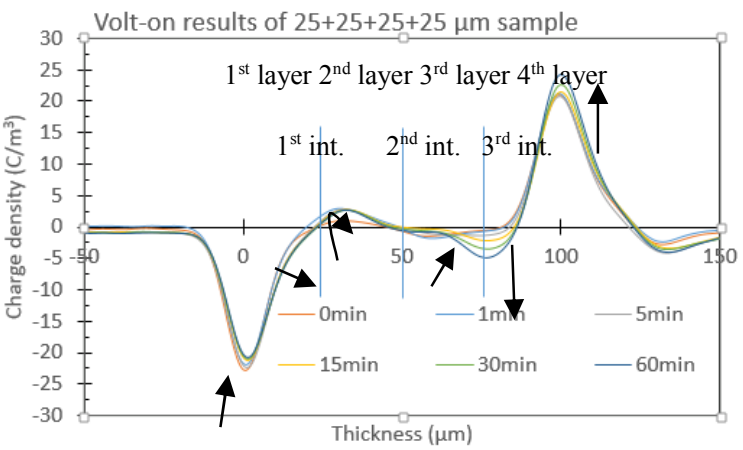

(c)

Fig. 4. Space charge profiles during volts-on in symmetrical configurations (a) two-layer, (b) three-layer and (c) four-layer.

Figure 4 (b) shows the space charge dynamics of the threelayer configuration during the voltage application process. Negative interfacial charges can be observed on the both interfaces. It seems that the injected negative charges could be partially blocked by the interface near the cathode, and accumulated in that region. The rest negative charges could propagate through that interface and then be blocked by the interface near the anode, leading to electric field enhancement in the PET layer contacted with the anode. The general space 
charge behaviour is dominated by negative charges, thus, the electric field near the cathode is reduced while increased near the anode.

In Figure 4 (c), the space charge behaviour is very complicated in four-layer configuration. Shortly after the voltage application, negative charges inject from the cathode and blocked by the first interface (accumulated in the PET layer contacted with the cathode side), meanwhile, positive charges can also be observed near the first interface, which accumulate in the second PET layer side. The amount of these accumulated positive charges grows quickly within the first minutes, then slightly decreases in the rest 59 minutes. It is believed the reduction is caused neutralization of injected negative charges from the cathode (propagate through the first interface). For the region near the second interface (the middle interface), only small amount of negative charge can be observed near the second interface in the third PET layer in the first 1 minute. After that, these charges reduced and move to third interface. Therefore, large amount of negative charges accumulate on the third interface, which is very similar in the three-layer configuration. In this case, the electric fields in the first interface and the PET layer contacted with the anode are enhanced.

Based on these initial study of charge dynamics in different sample configurations. It is difficulty to establish a direct link between charge formation and DC breakdown strength. More detailed studies are required: (i) behaviors and characteristics of positive and negatives charges in PET films; (ii) the different interfacial impacts on the positive and negative charges; (iii) the explanations for the negative charge dominated space charge dynamics occurred in the three-layer configuration; (iv) the explanation for the considerable negative charge accumulation on the interface near the anode in the three-layer and four-layer configurations; (v) the most important, the relationship between the space charge characteristics and the higher breakdown strength in these symmetrical configurations - it seems the electric field enhancement due to the accumulated charges is more severe in multilayer systems due to the presences of interfaces.

Although these initial results cannot fully answer the above questions, they still provide important guidance to further investigations:

(i) The impacts of the unsymmetrical electrodes (semicon anode and aluminum cathode) in the PEA system, which is different from the breakdown test;

(ii) The impacts of high electric fields on the space charge characteristics. At the present, the applied electric field in the space charge measurements is much lower than the breakdown strength. The space charge behaviour would be much more significant if the applied electric field is high [6];

(iii) The impacts of interfaces on the other electrical properties are also interesting, e.g. the DC conductivities for the different configurations, as the interfaces can partially block the propagation of charges as barriers.

(iv) The impacts of the film structure (place order) on the space charge characteristics in the unsymmetrical configurations; (v) The PEA measurement system with higher spatial resolution or rearrange the film thicknesses to allow a better observation of space charge profiles in thin film layers. The space charge profiles in $25 \mu \mathrm{m}$ films cannot be well recognized by the current measurement system.

\section{CONCLUSIONS}

In this paper, the DC breakdown strengths of five different configurations of PET films with an equal total thickness 100 $\mu \mathrm{m}$ have been investigated. It has been found that the breakdown strength is improved if the correct configuration is selected, e.g. symmetrical multilayer configurations. To further explore the mechanism behind this, space charge characteristics of these configurations haven been studied. The initial results have validated that large amount of fast moving charges exist in the PET film, which can be blocked by the presences of interfaces. The electric field distribution will be modified due to the accumulated charges on the interfaces, and resulting in field enhancement in the thinner layers, particularly in the unsymmetrical configuration. Further investigations are required to provide more information for better understandings.

\section{ACKNOWLEDGMENT}

The authors are grateful for the financial support from the technology project of State Grid Shaanxi Electric Power Company [5226KY16001G] and [2018GY-001].

\section{REFERENCES}

[1] G. Chen, M. Hao, Z. Xu., A. S. Vaughan, J. Cao and H. Wang, " Review of high voltage direct current cables", CSEE Journal of Power and Energy Systems, Vol. 1, No. 2, 9-2, 2015.

[2] J. Artbauer and J. Griac, "The Intrinsic Electric Strength of Polymers and Its Relation to the Structure Part I: Experimental", Acta Technica CSAV, No. 3, pp. 416-428, 1966.

[3] O.S. Gefle, S. M. Lebedev, Y. P. Pokholkov, I. Vitellas and D. P. Agoris, "Behaviour of multilayer PET polymer film insulation in high electric fields", IEE Proceedings - Science, Measurement and Technology, Vol. 151, No. 4, 2004, pp. 273-277.

[4] G. Chen, J. Zhao, S. Li and L. Zhong, "Origin of thickness dependent DC electrical breakdown in dielectrics". Appl. Phys. Lett.; 100: 222904-1 222904-4, 2012.

[5] J. Yoshida, T. Maezawa and H. Miyake, "Space charge accumulation and breakdown in LDPE and LDPE/MgO nano-composite under high dc stress at various temperatures", IEEE Conference on Electrical Insulation and Dielectric Phenomena, 2009.

[6] S. Kawano, K. Ishijawa, H. Miyake, Y. Tanaka, "Space charge accumulation characteristics in multilayered polyimide films under DC high stress", IEEE International Conference on Solid Dielectrics (ICSD), Italy, 2013.

[7] W. Weibull, "A statistical distribution function of wide applicability", $J$. Appl. Mech., Vol. 18, pp. 293-297, 1951.

[8] Z. An, J. Cang, X. Chen, and Y. Liu, "Method for investigating threshold field of charge injection at electrode/dielectric interfaces by space charge observation", Appl. Phys. Lett., 101: 172902-1 - 172902-4, 2012. 